\title{
Risk of neonatal drug withdrawal after intrauterine co-exposure to opioids and psychotropic medications: cohort study
}

\author{
Krista F Huybrechts, ${ }^{1}$ Brian T Bateman, ${ }^{1,2}$ Rishi J Desai, ${ }^{1}$ Sonia Hernandez-Diaz, ${ }^{3}$ Kathryn \\ Rough, ${ }^{1,3}$ Helen Mogun, ${ }^{1}$ Leslie S Kerzner, ${ }^{4}$ Jonathan M Davis, ${ }^{5}$ Megan Stover, ${ }^{6}$ Devan Bartels, ${ }^{7}$ \\ Jennifer Cottral, ${ }^{7}$ Elisabetta Patorno ${ }^{1}$
}

For numbered affiliations see end of article.

Correspondence to:

K F Huybrechts

khuybrechts@bwh.harvard.edu Additional material is published online only. To view please visit the journal online.

Cite this as: BMJ 2017;358:j3326 http://dx.doi.org/10.1136/bmj.j3326

Accepted: 05 July 2017

\section{ABSTRACT}

\section{OBJECTIVES}

To assess the impact of in utero co-exposure to psychotropic medications and opioids on the incidence and severity of neonatal drug withdrawal.

DESIGN

Observational cohort study.

\section{SETTING}

Nationwide sample of pregnancies in publicly insured women in the US, nested in the Medicaid Analytic eXtract (2000-10).

\section{PARTICIPANTS}

201275 pregnant women with public insurance who were exposed to opioids around the time of delivery and their liveborn infants.

\section{INTERVENTIONS}

In utero exposure to psychotropic medications, in particular antidepressants, atypical antipsychotics, benzodiazepines, gabapentin, and non-benzodiazepine hypnotics (Z drugs), with prescriptions filled within the same time window as prescriptions for opioids.

MAIN OUTCOME MEASURE

Diagnosis of neonatal drug withdrawal in infants exposed in utero to opioids and psychotropic medications compared with opioids alone.

\section{WHAT IS ALREADY KNOWN ON THIS TOPIC}

Neonatal drug withdrawal is common; in the US about every 25 minutes an infant is born with signs of drug withdrawal

Neonatal drug withdrawal is a well recognized complication of intrauterine exposure to illicit or prescription opioids, but other psychotropic medications can also cause signs of withdrawal

Psychotropic medications are often co-prescribed with opioids in pregnancy, and the use of both has increased significantly, raising concerns about an increase in the incidence and severity of neonatal drug withdrawal from potential drug-drug interactions, but these risks are not well understood

\section{WHAT THIS STUDY ADDS}

This large population based cohort study showed a 30-60\% increase in the risk of neonatal drug withdrawal associated with co-exposure to antidepressants, benzodiazepines, and gabapentin compared with opioids alone; no significant increase in risk was observed for atypical antipsychotics and Z drugs (nonbenzodiazepine hypnotics)

In utero exposure to psychotropic polypharmacy along with opioids is associated with a twofold increased risk of neonatal withdrawal

Withdrawal symptoms seem to be more severe in neonates with concurrent exposure to opioids and psychotropic medications compared with opioids alone

\section{RESULTS}

The absolute risk for neonatal drug withdrawal ranged from $1.0 \%$ in infants exposed in utero to prescription opioids alone to $11.4 \%$ for those exposed to opioids co-prescribed with gabapentin. Among neonates exposed in utero to prescription opioids, the relative risk adjusted for propensity score was 1.34 (95\% confidence interval 1.22 to 1.47$)$ with concomitant exposure to antidepressants, 1.49 (1.35 to 1.63 ) with benzodiazepines, 1.61 (1.26 to 2.06) with gabapentin, 1.20 (0.95 to 1.51) with antipsychotics, and 1.01 (0.88 to 1.15 ) with $Z$ drugs. In utero exposure to two or more psychotropic medications along with opioids was associated with a twofold increased risk of withdrawal $(2.05,1.77$ to 2.37$)$. The severity of the withdrawal seemed increased in neonates exposed to both opioids and psychotropic medications compared with opioids alone.

\section{CONCLUSIONS}

During pregnancy, the use of psychotropic medications in addition to prescription opioids is common, despite a lack of safety data. The current findings suggest that these drugs could further increase the risk and severity of neonatal drug withdrawal.

\section{Introduction}

Prescriptions for opioid analgesics in the US have increased more than threefold over the past two decades, ${ }^{12}$ and this rise also extends to prescriptions to pregnant women. About $14-22 \%$ of pregnancies in the US are complicated by exposure to prescription opioid medications. ${ }^{34}$ A strong increase in the prescription of opioids has also been observed in most European countries (such as Germany, Italy, Spain, Scandinavia, UK, the Netherlands) and Australia, though the number of prescriptions and defined daily doses remains much lower than in the US and Canada. ${ }^{5}$ Data from a population based registry in Norway showed that $6 \%$ of pregnant women filled at least one opioid prescription in 2004-06. ${ }^{6}$ Neonatal abstinence syndrome (NAS) is a well recognized complication of intrauterine exposure to opioid analgesics, with manifestations ranging from difficulties with feeding and sleeping to more severe complications including impaired thermoregulation, seizures, failure to thrive, and respiratory distress. ${ }^{7}$ From 2000 to 2012, hospital charges for this syndrome increased from about $\$ 190 \mathrm{~m}$ (about $£ 150 \mathrm{~m}$; €170m) to $\$ 1.5 \mathrm{bn} .{ }^{8}$ In the US, about every 25 minutes an infant is born with signs of drug withdrawal. ${ }^{89}$

Although neonatal drug withdrawal occurs most commonly in the context of antepartum illicit or prescription opioid use, other classes of medication-in 
particular psychotropic medications-can also cause signs of withdrawal. It is often difficult to determine whether the signs of withdrawal in the newborn are primarily the result of intrauterine exposure to opioids, other psychotropic medications (including nicotine), or a combination of both. The label for all antipsychotics was updated in 2011 to include the potential risk for withdrawal in newborns whose mothers were treated with these drugs during the third trimester of pregnancy, based on 69 cases reported in FDA's adverse event reporting system. ${ }^{10}$ Withdrawal has also been described in infants exposed antenatally to barbiturates, benzodiazepines, and antidepressants, ${ }^{7}$ and recently there have been reports of severe withdrawal from gabapentin. ${ }^{11}$ Psychotropic medications are often used by pregnant women who are also receiving an opioid, ${ }^{12-14}$ raising concerns about an increase in the incidence and severity of neonatal drug withdrawal from potential drug-drug interactions. ${ }^{14}$ Whereas intrauterine exposure to psychotropic medications has been used as a risk stratification factor, ${ }^{14}$ the risks of neonatal drug withdrawal in those exposed to opioids during the pregnancy with or without co-exposure to individual and combinations of psychotropic medications have not previously been examined. Little is therefore known about how this risk varies by the type of psychotropic medication or about the risks associated with psychotropic polypharmacy.

We assessed whether the risk of neonatal drug withdrawal in newborns exposed in utero to one or more psychotropic medications and opioid analgesics is greater and more severe than in those exposed to opioid analgesics alone in a cohort of publicly insured mother-infant dyads.

\section{Methods}

Data source and study cohort

The study cohort was nested in the nationwide Medicaid Analytic eXtract (MAX) for 2000-10. The MAX includes demographic and insurance enrollment information, medical visits, and admissions to hospital, inpatient or outpatient diagnoses and procedures, and prescriptions filled on an outpatient basis. We included all pregnancies in women aged 1255 who were linked to a liveborn infant (further details are available elsewhere ${ }^{15}$ ). Women were required to be eligible for Medicaid from 180 days before delivery through to one month after delivery. Infants were required to have the same coverage through Medicaid during the first month after birth or until death if this occurred sooner. All women were required to have filled at least one outpatient prescription for an opioid analgesic (see table A in appendix for a list of those included) during the 45 days before delivery. We chose this window to increase the likelihood of opioid exposure around the time of delivery.

\section{Psychotropic medications}

Women who also filled a prescription for psychotropic medication during the 45 days before delivery were considered as exposed. We chose this window to ensure intrauterine exposure to both opioids and psychotropic medications near or at the time of delivery, which is relevant for neonatal drug withdrawal. We distinguished between five different exposure groups: antidepressants, atypical antipsychotics (hereafter antipsychotics), gabapentin (included because of its mood stabilizing properties), benzodiazepines, and non-benzodiazepine hypnotics (hereafter $\mathrm{Z}$ drugs) (table B in the appendix). In secondary analyses, we also examined the risk associated with exposure to one or more of the psychotropic medications found to be associated with an increased risk of withdrawal in the primary analyses. The reference group consisted of women who received opioids but who did not fill an outpatient prescription for the psychotropic medication of interest during the 180 days before delivery.

\section{Neonatal drug withdrawal}

Neonatal drug withdrawal was defined based on the presence of ICD-9 (international classification of diseases, ninth revision) code $779.5 \mathrm{x}$ in maternal or infant claims from delivery to one month after delivery. We analyzed the maternal record as Medicaid claims are sometimes recorded under the mother before the infant's eligibility has been processed. ${ }^{11}$ The outcome definition was validated in the subset of study participants who received their obstetrical care in the Partners HealthCare system. We randomly selected a sample of cases of neonatal drug withdrawal $(n=50)$ that occurred in the absence of exposure to prescription opioids (for example, withdrawal from heroin, illicit opioids, methadone) and all cases of withdrawal that occurred with known exposure to prescription opioids $(n=7)$ and retrieved their electronic medical records at the delivery hospital. Two reviewers (DB and JC), blinded to the exposure status, adjudicated these cases; discrepancies were resolved by consensus (four of 50 cases with prescription opioids, none of the seven cases without prescription opioids). We estimated a positive predictive value of $91 \%$ (95\% confidence interval $82 \%$ to $97 \%$ ) overall and $100 \%$ (65\% to $100 \%$ ) in the subset of infants with intrauterine exposure to prescribed opioids.

\section{Covariates}

The specific compound, form, and dose of opioid are important potential confounding variables. We expressed the dose as the total amount of prescription opioids accumulated during the last 45 days of pregnancy in oral morphine equivalents (see table A in appendix). ${ }^{16-18}$ Additional covariates assessed as potential confounders included calendar year, demographics (age, race/ethnicity), obstetric characteristics (multiple gestation, preterm delivery, pre-eclampsia), treatment indications (pain, psychiatric diagnoses), indicators of substance abuse or dependence, concomitant medications, and general markers of disease burden (intensity of healthcare use). We also assessed diagnoses of alcohol or drug abuse or dependence, tobacco smoking, methadone, 
and use of buprenorphine, naloxone, and naltrexone. Other than the psychotropic exposures of interest, we considered the concomitant use of other anxiolytics, barbiturates, other hypnotics, stimulants, primidone, phenobarbital, and pregabalin, all of which have been associated with withdrawal in adults. ${ }^{19}$ General markers of disease burden included the obstetric morbidity index, ${ }^{20}$ number of distinct prescriptions, number of distinct diagnoses, and number of outpatient visits, hospital admissions, and emergency room visits. ${ }^{21}$ All variables were assessed from 180 days before delivery through to delivery, except for measures of intensity of healthcare use, which were assessed from 180 to 90 days before delivery to avoid these measures being affected by early awareness of possible late pregnancy complications (table $\mathrm{C}$ in the appendix shows a complete list of covariates).

\section{Data analyses}

We described baseline characteristics in women exposed and unexposed to different psychotropic medications and estimated the absolute risk of neonatal drug withdrawal for each exposure group, as well as the unadjusted risk ratio with its 95\% confidence interval for each exposure contrast. In the first adjusted analysis, we accounted for the specific opioid compound (that is, generic name and form (immediate release or extended release) as listed in table $\mathrm{A}$ in the appendix) and dose expressed in morphine equivalents, which is expected to be the most important confounder. In the second adjusted analysis, we also accounted for additional potential confounders, as described above (table $\mathrm{C}$ in the appendix). For each of the two levels of analysis, we used propensity scores as a summary confounding score estimated using logistic regression given the large number of prespecified variables. We included only main effects in the models. We restricted the population to observations within a propensity score range that was common to both exposed and unexposed women and created 25 equally sized strata based on the distribution among the women exposed to psychotropics. ${ }^{22}$ The unexposed observations (that is, exposed only to opioid) were weighted with the distribution of the treated among propensity score strata. We assessed balance of baseline characteristics in this weighted population, which considers potential confounding variables, using the standardized mean difference. An absolute standardized mean difference over 0.1 was considered as evidence of residual imbalance. Adjusted effects were estimated with generalized linear models (SAS Proc Genmod with a weight statement and log link function).

\section{Sensitivity analyses}

As we might not have accounted for all potential confounders available in our data using prespecified covariates, we conducted high dimensional propensity score analyses that included 200 empirically defined covariates, in addition to the investigator defined covariates, to account for potential residual confounding. ${ }^{23}$ We then varied the exposure definition for propensity score adjusted analyses by expanding the exposure window for both opioids and psychotropics from 45 to 90 days before delivery to evaluate the potential risk associated with exposure earlier in pregnancy and by requiring two or more prescriptions for a psychotropic medication to be filled during the 90 days before delivery, at least one of which had to be filled during the last 45 days to reduce the likelihood of misclassification of exposure. Subclasses of antidepressant medications (selective serotonin reuptake inhibitors (SSRIs), selective norepinephrine reuptake inhibitors, tricyclic antidepressants, bupropion, other antidepressants) were examined as well as the most commonly used SSRIs (citalopram, escitalopram, fluoxetine, paroxetine, sertraline); this was feasible given the large number of women taking antidepressants in late pregnancy.

\section{Exploratory analyses: severity of withdrawal}

As we did not have Finnegan scores $^{24}$ available, we explored whether proxies for the severity of withdrawal symptoms in neonates with in utero exposure to one or more of the psychotropic medications were different compared with those exposed only to opioids. Proxy severity indicators included a recorded diagnosis in the first 30 days of life indicating feeding difficulties (ICD9 779.3x), respiratory symptoms (ICD-9 769.xx-770. $\mathrm{xx}$ ), or signs of seizures (ICD-9 779.0x and 780.3x). We also evaluated a composite outcome defined as the presence of any of these three severity indicators and the neonate's length of hospital stay. Adjusted analyses used the same set of covariates and analytic approach as the main analyses.

\section{Patient involvement}

No patients were involved in setting the research question or the outcome measures, nor were they involved in developing plans for design or implementation of the study. No patients were asked to advise on interpretation or writing up of results. There are no plans to disseminate the results of the research to study participants or the relevant patient community.

\section{Results}

The cohort included 201275 pregnancies in women with exposure to prescription opioids in the 45 days before delivery. Among those, 14183 women (7.0\%) also filled a prescription for an antidepressant, $993(0.5 \%)$ for an antipsychotic, 5361 (2.7\%) for a benzodiazepine, $501(0.3 \%)$ for gabapentin, and $10105(5.0 \%)$ for a $\mathrm{Z}$ drug. Women exposed to psychotropic medications differed from those who were not exposed on most of the baseline characteristics. Exposed women were older, were more likely to be white, had more pain and psychiatric diagnoses, used more other psychotropic medications, more commonly had diagnoses of substance abuse or dependence, and had a higher burden of comorbidity. They also tended to have a higher cumulative dose of opioids during the last 45 days of their pregnancy, which was 


\begin{tabular}{|c|c|c|c|c|c|c|c|c|c|}
\hline \multirow[b]{2}{*}{ Characteristics } & \multicolumn{3}{|l|}{ Unadjusted } & \multicolumn{3}{|l|}{ PS adjustedt } & \multicolumn{3}{|c|}{ hdPS adjustedt } \\
\hline & $\begin{array}{l}\text { Gabapentin } \\
\text { and opioids }\end{array}$ & Opioids only & SMD & $\begin{array}{l}\text { Gabapentin } \\
\text { and opioids }\end{array}$ & Opioids only & SMD & $\begin{array}{l}\text { Gabapentin } \\
\text { and opioids }\end{array}$ & Opioids only & SMD \\
\hline Total & 501 & 200204 & - & 501 & 200204 & - & 491 & 186224 & - \\
\hline Antipsychotics & $51(10.18)$ & $2024(1.01)$ & 0.41 & $51(10.18)$ & 25209 (12.59) & -0.08 & $47(9.57)$ & $17271(9.27)$ & 0.01 \\
\hline Benzodiazepines & $129(25.75)$ & $9134(4.56)$ & 0.62 & $129(25.75)$ & $56823(28.38)$ & -0.06 & $125(25.46)$ & $47858(25.7)$ & -0.01 \\
\hline Antidepressants & $237(47.31)$ & $26942(13.46)$ & 0.79 & $237(47.31)$ & $87592(43.75)$ & 0.07 & $230(46.84)$ & $87908(47.21)$ & -0.01 \\
\hline$Z$ drugs & $80(15.97)$ & $12879(6.43)$ & 0.31 & $80(15.97)$ & $32711(16.34)$ & -0.01 & $77(15.68)$ & $29567(15.88)$ & -0.01 \\
\hline \multicolumn{10}{|l|}{ Demographics } \\
\hline Mean (SD) age (years) & $28.33(5.12)$ & $24.6(5.6)$ & 0.70 & $28.33(5.72)$ & $27.09(5.59)$ & 0.22 & $28.29(5.59)$ & $28.41(5.61)$ & -0.02 \\
\hline White race & $396(79.04)$ & $132000(65.93)$ & 0.30 & $396(79.04)$ & $155803(77.82)$ & 0.03 & $388(79.02)$ & $149033(80.03)$ & -0.02 \\
\hline Black race & $62(12.38)$ & $42132(21.04)$ & -0.23 & $62(12.38)$ & 23807 (11.89) & 0.01 & $60(12.22)$ & $21297(11.44)$ & 0.02 \\
\hline Hispanic race & $13(2.59)$ & $12499(6.24)$ & -0.18 & $13(2.59)$ & $7424(3.71)$ & -0.06 & $13(2.65)$ & $4953(2.66)$ & 0.00 \\
\hline \multicolumn{10}{|l|}{ Obstetric characteristics } \\
\hline Preterm birth & $117(23.35)$ & $26235(13.1)$ & 0.27 & $117(23.35)$ & $44272(22.11)$ & 0.03 & $115(23.42)$ & $43094(23.14)$ & 0.01 \\
\hline Pre-eclampsia & $43(8.58)$ & $10057(5.02)$ & 0.14 & $43(8.58)$ & $15609(7.8)$ & 0.03 & $42(8.55)$ & $15540(8.34)$ & 0.01 \\
\hline \multicolumn{10}{|l|}{ Pain } \\
\hline Neuropathic & $112(22.36)$ & $7948(3.97)$ & 0.56 & $112(22.36)$ & $33883(16.92)$ & 0.14 & $105(21.38)$ & $39978(21.47)$ & 0.00 \\
\hline Musculoskeletal & $188(37.52)$ & $27965(13.97)$ & 0.56 & $188(37.52)$ & $72918(36.42)$ & 0.02 & $180(36.66)$ & $68202(36.62)$ & 0.00 \\
\hline Back or neck & $227(45.31)$ & $46619(23.29)$ & 0.48 & $227(45.31)$ & 87901 (43.91) & 0.03 & $220(44.81)$ & $84345(45.29)$ & -0.01 \\
\hline Fibromyalgia & $57(11.38)$ & $3164(1.58)$ & 0.41 & $57(11.38)$ & $21526(10.75)$ & 0.02 & $55(11.2)$ & $20491(11)$ & 0.01 \\
\hline Migraine/headache & $154(30.74)$ & $31888(15.93)$ & 0.36 & $154(30.74)$ & $57608(28.77)$ & 0.04 & $151(30.75)$ & $57980(31.13)$ & -0.01 \\
\hline Other & $45(8.98)$ & $3231(1.61)$ & 0.33 & $45(8.98)$ & $20054(10.02)$ & -0.04 & $43(8.76)$ & $15832(8.5)$ & 0.01 \\
\hline \multicolumn{10}{|l|}{ Psychiatric diagnoses } \\
\hline Bipolar disorder & $56(11.18)$ & $3595(1.8)$ & 0.39 & $56(11.18)$ & $27138(13.56)$ & -0.07 & $51(10.39)$ & $19688(10.57)$ & -0.01 \\
\hline Schizophrenia & $2(0.4)$ & $294(0.15)$ & 0.05 & $2(0.4)$ & $1986(0.99)$ & -0.07 & $2(0.41)$ & $763(0.41)$ & 0.00 \\
\hline Depression & $81(16.17)$ & $11458(5.72)$ & 0.34 & $81(16.17)$ & $38779(19.37)$ & -0.08 & $77(15.68)$ & $28113(15.1)$ & 0.02 \\
\hline Anxiety & $90(17.96)$ & $8463(4.23)$ & 0.45 & $90(17.96)$ & $43230(21.59)$ & -0.09 & $86(17.52)$ & $32513(17.46)$ & 0.00 \\
\hline Sleep disorder & $13(2.59)$ & $1553(0.78)$ & 0.14 & $13(2.59)$ & $7506(3.75)$ & -0.07 & $12(2.44)$ & $4666(2.51)$ & 0.00 \\
\hline \multicolumn{10}{|c|}{ Indicators of substance abuse or dependence } \\
\hline Alcohol abuse or dependence & $8(1.6)$ & $1004(0.5)$ & 0.11 & $8(1.6)$ & $3423(1.71)$ & -0.01 & $8(1.63)$ & $2916(1.57)$ & 0.01 \\
\hline Drug abuse or dependenceł & $40(7.98)$ & $4771(2.38)$ & 0.25 & $40(7.98)$ & $18337(9.16)$ & -0.04 & $38(7.74)$ & $14381(7.72)$ & 0.00 \\
\hline Tobacco use & $95(18.96)$ & $19888(9.93)$ & 0.26 & $95(18.96)$ & $37395(18.68)$ & 0.01 & $93(18.94)$ & $34393(18.47)$ & 0.01 \\
\hline \multicolumn{10}{|l|}{ Specific opioids } \\
\hline $\begin{array}{l}\text { Mean (SD) morphine equiva- } \\
\text { lents (mg) }\end{array}$ & 1169 (1809) & $326(2451)$ & 0.39 & $1169(7419)$ & $1497(2449)$ & -0.06 & $1116(3703)$ & $1164(2287)$ & -0.02 \\
\hline Codeine & $130(25.95)$ & $95168(47.54)$ & -0.46 & $130(25.95)$ & $53727(26.84)$ & -0.02 & $128(26.07)$ & $46789(25.13)$ & 0.02 \\
\hline Hydrocodone & $267(53.29)$ & $89850(44.88)$ & 0.17 & $267(53.29)$ & $100448(50.17)$ & 0.06 & $263(53.56)$ & $100418(53.92)$ & -0.01 \\
\hline Oxycodone§ & $134(26.75)$ & $24820(12.4)$ & 0.37 & $134(26.75)$ & $49392(24.67)$ & 0.05 & $129(26.27)$ & $48244(25.91)$ & 0.01 \\
\hline Other opioids & $44(8.78)$ & 3949 (1.97) & 0.31 & $44(8.78)$ & $18112(9.05)$ & -0.01 & $41(8.35)$ & $15547(8.35)$ & 0.00 \\
\hline
\end{tabular}

*Table illustrates balance of selected baseline characteristics for gabapentin. Similar tables for the other psychotropic medications and comprehensive table for gabapentin are provided in tables D-J in appendix.

tTo account for PS/hdPS, untreated (opioids only) observations were weighted with distribution of treated (gabapentin and opioids) among PS/hdPS strata. Observations in non-overlapping areas of PS/hdPS distribution were dropped.

‡Drug abuse or dependence, methadone, methadone extended release, methadone maintenance, buprenorphine, naloxone, naltrexone. $\S$ Immediate release/short acting (IR/SA) and extended release/long acting (ER/LA).

IButorphanol (IR/SA), fentanyl (IR/SA and ER/LA), hydromorphone (IR/SA), meperidine (IR/SA), morphine (IR/SA and ER/LA), oxymorphone (IR/SA and ER/LA), pentazocine (IR/SA), propoxyphene (IT/SA), tapentadol (IR/SA), tramadol (ER/LA).

most prominent for users of benzodiazepines (mean dose $1352 \mathrm{mg} v 291 \mathrm{mg}$ oral morphine equivalents) and gabapentin (1169 mg $v 326 \mathrm{mg}$ ). The difference in baseline characteristics was less pronounced for women exposed to $\mathrm{Z}$ drugs (table 1, fig 1, and tables D-J in the appendix).

The absolute risk for neonatal drug withdrawal was substantially higher among women who were coexposed to opioids and psychotropic medications than among women exposed to opioids alone. Absolute risk of withdrawal in neonates ranged from $2.3 \%$ among women exposed to $\mathrm{Z}$ drugs to $11.4 \%$ among women exposed to gabapentin. The risk among women exposed to prescription opioids alone was around $1 \%$, with small variations given the different exclusions made in each exposure contrast (table 2).
The unadjusted relative risk was 3.48 (95\% confidence interval 3.15 to 3.84) for antidepressants, 5.42 (4.28 to 6.85) for antipsychotics, 7.43 (6.71 to 8.23) for benzodiazepines, 9.08 (7.09 to 11.63) for gabapentin, and 1.87 (1.63 to 2.13) for Z drugs. The strength of the associations was much attenuated after adjustment for specific opioid compound, form (that is, extended versus immediate release), and cumulative dose (fig 2, table $\mathrm{K}$ in the appendix).

Infully adjusted analyses, the baseline characteristics were well balanced (fig 1, table 1, and tables D-J in the appendix). The risk of withdrawal was increased for antidepressants (1.34, 95\% confidence interval 1.22 to 1.47$)$, benzodiazepines $(1.49,1.35$ to 1.63$)$, and gabapentin (1.61, 1.26 to 2.06), and, to a lesser degree, for antipsychotics $(1.20,0.95$ to 1.51$)$, but not 


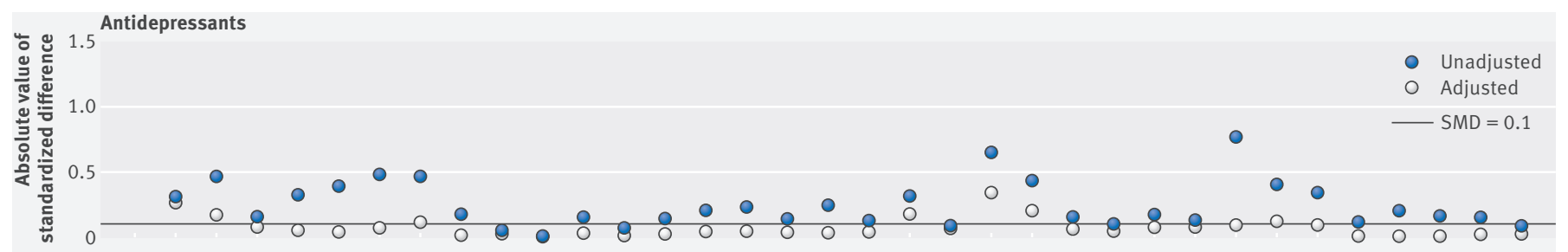

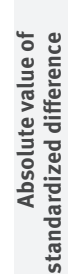

Benzodiazepines

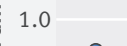

营

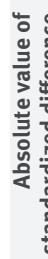

Gabapentin
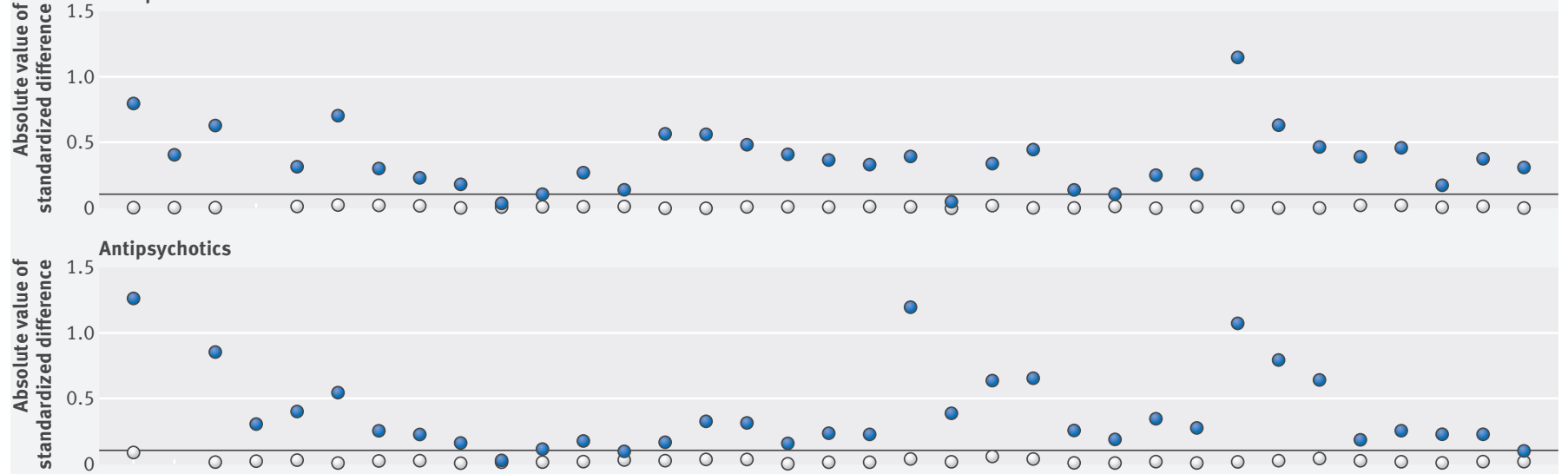

它

$Z$ drugs

1.0

문.

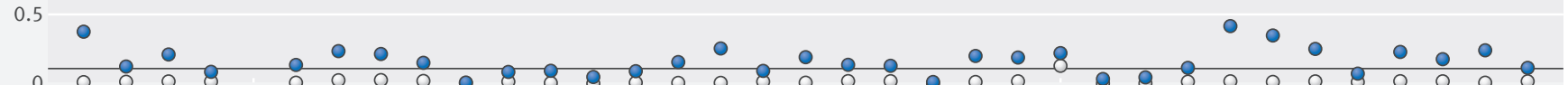

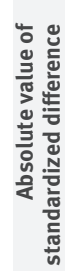

1 psychotropic

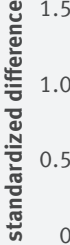

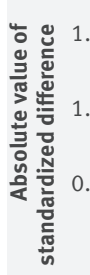
$\geq 2$ psychotropics
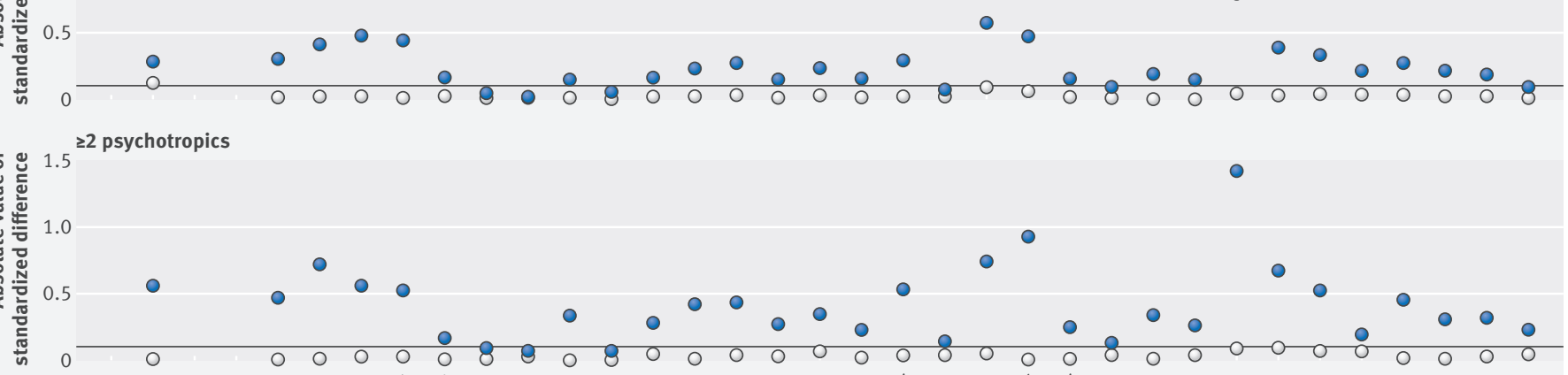

1.0
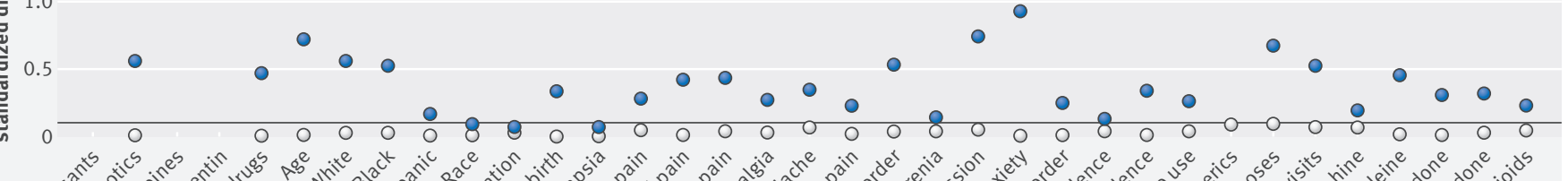

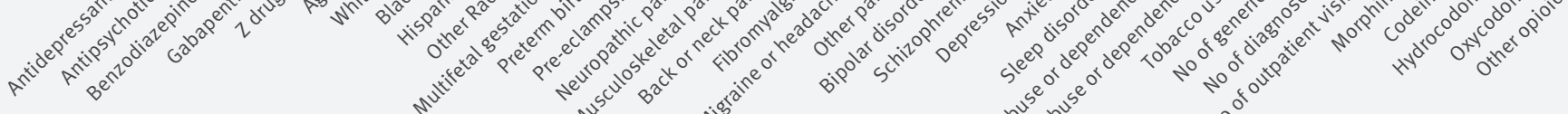
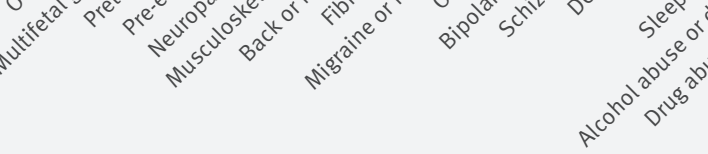

Characteristics

Fig 1 | Absolute standardized mean difference illustrating balance in baseline characteristics before and after adjustment for high dimensional propensity score. SMD $<0.1$ indicates characteristics are well balanced 


\begin{tabular}{|c|c|c|c|c|}
\hline & \multicolumn{2}{|c|}{ Opioids + psychotropic medications } & \multicolumn{2}{|l|}{ Opioids alone } \\
\hline & Cases/total & $\operatorname{Risk}(/ 100)(95 \% \mathrm{Cl})$ & Cases/total & Risk (/100) $(95 \% \mathrm{Cl})$ \\
\hline Antidepressants & $495 / 14183$ & 3.49 (3.19 to 3.79$)$ & $1743 / 173841$ & 1.00 (0.96 to 1.05$)$ \\
\hline Antipsychotics & $67 / 993$ & $6.75(5.19$ to 8.31$)$ & $2481 / 199151$ & 1.25 (1.20 to 1.29$)$ \\
\hline Benzodiazepines & $413 / 5361$ & 7.70 (6.99 to 8.42$)$ & 1989/191863 & 1.04 (0.99 to 1.08$)$ \\
\hline Gabapentin & $57 / 501$ & 11.38 (8.60 to 14.16$)$ & $2509 / 200204$ & 1.25 (1.20 to 1.30$)$ \\
\hline Z drugs & $229 / 10105$ & 2.27 (1.98 to 2.56$)$ & $2286 / 188216$ & 1.21 (1.17 to 1.26$)$ \\
\hline 1 psychotropic* & $612 / 16524$ & $3.70(3.42$ to 4.00$)$ & $1423 / 168086$ & 0.85 (0.80 to 0.89$)$ \\
\hline$\geq 2$ psychotropics* & $172 / 1737$ & $9.90(8.56$ to 11.37$)$ & $1423 / 168086$ & 0.85 (0.80 to 0.89$)$ \\
\hline
\end{tabular}

for Z drugs (1.01, 0.88 to 1.15). Exposure to any single additional psychotropic medication (antidepressants or benzodiazepines or gabapentin) was associated with a $37 \%$ increased risk of withdrawal $(1.37,1.26$ to 1.49 ) in adjusted analyses. Exposure to two or more of these medications was associated with a doubling of the risk $(2.05,1.77$ to 2.37 ; fig 2 , table $\mathrm{K}$ in the appendix)

\section{Sensitivity analyses}

Our findings were corroborated in analyses adjusted for high dimensional propensity score, which showed an increased risk for antidepressants, benzodiazepines, and gabapentin. The results for any one or multiple psychotropics were upheld as well. We found no association for antipsychotics and $\mathrm{Z}$ drugs with relative risks of 1.03 and 1.00 , respectively (fig 2 , table $\mathrm{K}$ in the appendix). The balance in baseline characteristics was

Partially adjusted
Antidepressants
Antipsychotics
Benzodiazepines
Gabapentin
Z drugs
1 psychotropic
z2 psychotropics

Fig 2 | Adjusted relative risk of neonatal drug withdrawal according to maternal exposure to psychotropic medications in addition to prescription opioids, according to level of adjustment for confounding. Medicaid Analytic eXtract, 2000-10 further improved with high dimensional propensity score stratification compared with propensity score stratification, in particular for antipsychotics and gabapentin (fig 1). Extension of the exposure window to 90 days before delivery and a requirement for two or more prescriptions for a psychotropic medication to be filled during the 90 days before delivery did not change the findings either (table $\mathrm{L}$ in the appendix). All antidepressant subclasses and specific SSRIs seemed to be associated with a small to modest increase in risk, but some of the associations were imprecisely estimated (table $\mathrm{M}$ in the appendix).

\section{Exploratory analyses}

Among infants with a diagnosis of drug withdrawal, intrauterine exposure to antidepressants, benzodiazepines, or gabapentin in addition to opioids seemed to be associated with an increase in the severity of symptoms of withdrawal assessed with proxy indicators of severity, compared with exposure to opioids alone, after adjustment for opioid dose and other potential confounding variables (1.16, 95\% confidence interval 1.06 to 1.27$)$. The effect was most pronounced for seizures $(1.68,1.03$ to 2.72 ; table 3 ). The mean length of stay in hospital was increased by one day, from 13 to 14 days (mean difference 1.13 days, $95 \%$ confidence interval -0.03 to 2.28 ; $\mathrm{P}<0.06$ ).

\section{Discussion}

\section{Statement of principal findings}

In this cohort of 201275 pregnancies in women who used prescription opioids close to delivery, we observed a $30-60 \%$ increase in the risk of neonatal drug withdrawal associated with co-exposure to antidepressants, benzodiazepines, and gabapentin. Exposure to two or more of these psychotropic medications was associated with a doubling of the risk. We found no significant increase in risk with atypical antipsychotics and $\mathrm{Z}$ drugs (non-benzodiazepine hypnotics). The severity of the withdrawal symptoms seemed to increase with concurrent exposure to psychotropic medications and opioids.

\section{Comparison with other studies}

Psychotropic medications, notably benzodiazepines and SSRIs, have been identified as exposures that increase the incidence and severity of neonatal drug withdrawal in studies of pregnant women with 


\begin{tabular}{|c|c|c|c|c|c|}
\hline \multirow{2}{*}{ Severity indicator } & \multicolumn{2}{|c|}{$\begin{array}{l}\text { Psychotropic medications* }+ \\
\text { opioids }\end{array}$} & \multicolumn{2}{|c|}{ Opioids alone } & \multirow[b]{2}{*}{$\mathrm{RR}(95 \% \mathrm{Cl})$} \\
\hline & Events & No of cases & Events & No of cases & \\
\hline \multicolumn{6}{|l|}{ Unadjusted } \\
\hline Signs of seizures & 35 & 784 & 46 & 1423 & 1.38 (0.90 to 2.12) \\
\hline Composite $†$ & 428 & 784 & 653 & 1423 & $1.19(1.09$ to 1.30$)$ \\
\hline \multicolumn{6}{|c|}{ Adjusted for propensity score } \\
\hline Respiratory symptoms & 286 & 717 & 439 & 1332 & $1.16(1.04$ to 1.31$)$ \\
\hline Feeding difficulties & 184 & 717 & 290 & 1332 & $1.08(0.93$ to 1.27$)$ \\
\hline
\end{tabular}

addiction. In a study of 450 women who misused drugs and were prescribed substitute methadone, maternal use of benzodiazepines significantly increased the likelihood of neonatal drug withdrawal requiring treatment (odds ratio 1.73, 95\% confidence interval 1.17 to 2.55) in univariate analyses. Maternal use of SSRIs was associated with a small non-significant increase in risk $(1.30,0.63$ to 2.70$){ }^{25}$ Similarly, a small study in an urban drug treatment center found that nearly twice as many newborns (83\% v 42\%) of women exposed to methadone and other illicit substances including benzodiazepines $(n=19)$ received pharmacotherapy for more severe neonatal drug withdrawal than newborns born to women receiving methadone only $(n=18)$. There was no difference, however, in the number of days of pharmacotherapy required to treat the withdrawal, indicating that while there was a difference in the incidence of withdrawal, there was no difference in severity among affected infants. ${ }^{26}$ In contrast, a study of infants with drug withdrawal born at Boston Medical Center in 200309 found that maternal use of prescribed methadone for opiate addiction and benzodiazepines $(n=56)$ compared with methadone alone $(n=158)$ increased the length of stay for infants with drug withdrawal by six days. Maternal use of methadone and SSRIs $(n=51)$ increased the length of stay by four and a half days. ${ }^{27}$ Based on these findings, intrauterine exposure to psychotropic medications has been used as a risk stratification factor in a study examining the risk of neonatal drug withdrawal with exposure to prescription opioids. ${ }^{14}$ We built on this work by examining the absolute and relative risk and the severity of withdrawal with co-exposure to specific psychotropics and combinations of psychotropic medications.

\section{Strengths and limitations}

The strengths of this study relate to both internal and external validity. The large size of the exposed groups allows for the estimation of the effect sizes with good precision, reducing the risk of random error. The richness of the source data allows for adjustment for a broad range of (proxies for) potential confounding variables, reducing the risk of confounding bias. As all exposure and covariate information is recorded before the occurrence of the withdrawal, there is no concern for differential recording. The study cohort is drawn from a nationwide sample of publicly insured pregnant women, and almost half of all pregnant women in the US are publicly insured. As there is no reason to believe that the underlying biological relations would be different in this study population compared with other populations of pregnant women, we should be able to generalize from our observations to the broader experience of pregnancies inside the US and elsewhere, where the use of prescription opioids is also on the rise. ${ }^{28}$

Several limitations should also be noted. Given the nature of the data, meconium or cord toxicology is not available to document fetal exposure. As several of the opioids included in the study can be used on an as needed basis, their use might not lead to relevant fetal exposure. This results in a lower overall risk of neonatal withdrawal than that seen in women with substance use disorders or addiction or in women in medication assisted treatment programs, as has previously been documented. ${ }^{14}$ It should be noted, however, that our objective was to assess the risk of withdrawal in neonates of women who were coprescribed opioid analgesics and other psychotropics. The fact that in many of these women fetal exposure could be minimal and will not lead to withdrawal is an important finding in itself. ICD-9 $779.5 \mathrm{x}$ is the main code used to record drug withdrawal syndrome in the infant of an opioid exposed mother and does not enable us to distinguish a synergistic effect between opioids and psychotropics (that is, an increase in the risk of opioid withdrawal) from an independent effect of psychotropics (that is, risk of withdrawal from psychotropic medications themselves). In clinical practice, however, it is also challenging to differentiate whether the withdrawal symptoms are attributable to opioids, other medications, or a combination of the two, making the management of these infants challenging, as illustrated in the case reported in the appendix. Although we observed a doubling of the risk in infants who had been exposed to two or more 
psychotropic medications in addition to prescription opioids, we cannot exclude the possibility that pediatricians are more likely to diagnose withdrawal in infants with known exposure to polypharmacy. It should be acknowledged that in some cases the constellation of neonatal symptoms after in utero exposure to SSRIs could be better explained by potential toxicity associated with increased serotonin concentrations rather than withdrawal (attributable to a relative hyposerotonergic state). ${ }^{7}$ We were not able to evaluate the risk of withdrawal in infants of women on maintenance treatment with buprenorphine or methadone because such treatment is not captured comprehensively in our data, but this is unlikely to confound the analyses given our restriction to a population with prescription opioids. Our data source did not contain a quantitative measure of severity such as the Finnegan score. ${ }^{24}$ We therefore explored the effect of exposure on the severity of withdrawal using indirect proxies, which are less sensitive to changes in severity than a direct quantitative measure and which might actually reflect other co-morbidities. Likewise, length of stay as documented in healthcare use data is an imperfect measure of severity as it can be affected by unrelated medical conditions or social concerns such as the need to arrange for foster care before discharge. In some parts of the US, infants are discharged home while they are still being weaned off opioids or other medications, which would result in an underestimation of the severity of withdrawal. The relatively short hospital length of stay and the one day increase because of concomitant exposure to psychotropics should be interpreted in that context. Unfortunately, information on medications used to treat the neonate's withdrawal symptoms in hospital was not available. We also did not have information on breastfeeding, which could reduce the severity of withdrawal. $^{29}$

Other limitations are those characteristic of drug safety studies that use large administrative databases or nationwide registries. Dispensing of a medication does not necessarily imply that it was actually taken or was taken as prescribed. Given that we observed an increased risk for several of the exposure groups, this is less of a concern as misclassification of the exposure is expected to bias towards the null. Despite our focus on prescription opioids, the possibility remains that the level of exposure could have been underestimated if some women also took opioids illicitly or overestimated if some women, for example, sold or passed on their opioids and therefore did not take them. In that regard it is reassuring that we did not observe an increased risk for infants of women exposed to antipsychotics as the likelihood of abuse is high in this group of more severely ill women. As gabapentin is often used off label as an analgesic, its use could be associated with more severe pain and illicit opioid use, which could lead to residual confounding. Sensitivity analyses, however, indicate that the strength of the association between unmeasured illicit opioid use and both gabapentin exposure and neonatal drug withdrawal would have to be unrealistically high for confounding to fully explain the observed association, especially as we adjusted for pain indications and indicators of substance abuse and dependence (fig B in the appendix). Some (milder) cases might have been missed if physicians did not associate an ICD-9 diagnosis with symptoms such as crying, irritability, jitteriness, and restlessness. As long as the specificity of the outcome definition is high (as indicated by the results of our internal validation study) and the sensitivity is non-differential between exposed and unexposed pregnancies, however, the relative risk is unbiased. Finally, we were unable to examine the risk associated with the first generation typical antipsychotics given the small number of exposed pregnancies $(n=119)$.

\section{Interpretation and clinical implications}

In conclusion, our findings suggest that among women using prescription opioids during pregnancy, co-exposure to antidepressants, benzodiazepines, and gabapentin might be associated with an increased risk of drug withdrawal in the neonate. The findings for gabapentin are consistent with earlier observations of symptoms of withdrawal in the adult population after abrupt cessation of high dose gabapentin ${ }^{3031}$ and observations in a recently published case series of 19 infants exposed to both opioids and gabapentin prenatally. ${ }^{32}$ This is especially concerning as gabapentin is being prescribed more often for control of pain. It has also been suggested that gabapentin is sometimes used illicitly to enhance the "high" associated with methadone treatment.

Chronic pain management is a serious global health priority, with chronic pain affecting around $20 \%$ of the population. Women are affected more often than men. The use of prescription opioids has grown significantly over the past two decades, including among pregnant women. The increased risks of neonatal drug withdrawal in those born to women concomitantly using opioids and antidepressants, benzodiazepines, or gabapentin suggest that clinicians should be cautious in prescribing these medications together in late pregnancy and in prescribing psychotropic medications to women with known or suspected illicit opioid use. Because pain and mental health conditions often occur together, co-exposure will be unavoidable in many instances. In those cases, our findings might be helpful in risk stratification for exposed infants. Our findings also imply that it will be important for neonatologists and pediatricians to rethink treatment protocols for infants born to women who were prescribed multiple drugs during their pregnancy.

\section{AUTHOR AFFILIATIONS}

${ }^{1}$ Division of Pharmacoepidemiology and Pharmacoeconomics, Department of Medicine, Brigham and Women's Hospital and Harvard Medical School, Boston, MA, USA

${ }^{2}$ Department of Anesthesiology, Perioperative and Pain Medicine, Brigham and Women's Hospital and Harvard Medical School, Boston, MA, USA 
${ }^{3}$ Department of Epidemiology, Harvard T.H. Chan School of Public Health, Boston, MA, USA

${ }^{4}$ Department of Pediatrics, Massachusetts General Hospital for Children and Harvard Medical School, Boston, MA, USA

${ }^{5}$ Department of Pediatrics, The Floating Hospital at Tufts Medical Center and the Tufts Clinical and Translational Science Institute, Tufts University , Boston, MA, USA

${ }^{6}$ Department of Obstetrics and Gynecology, Tufts Medical Center and Tufts University School of Medicine, Boston, MA, USA

${ }^{7}$ Department of Anesthesia, Critical Care, and Pain Medicine, Massachusetts General Hospital and Harvard Medical School, Boston, MA, USA

We thank Mengdong He and Sara Dejene, who helped with the implementation of the outcome validation study.

Contributors: KFH conceptualized and designed the study, carried out the analyses, and drafted the initial manuscript. BTB, RID, SH-D, $\mathrm{KR}$, and EP conceptualized and designed the study, critically reviewed the results of analyses, and reviewed and revised the manuscript. HM carried out the analyses and reviewed and revised the manuscript. LSK, JMD, and MS critically reviewed the results of analyses and reviewed and revised the manuscript. DB and JC adjudicated the neonatal withdrawal cases in the outcome validation study, critically reviewed the analyses results, reviewed and revised the manuscript. All authors approved the final manuscript as submitted. KFH is guarantor.

Funding: This study was supported by an R01 grant (R01 MH100216) from the National Institute of Mental Health. KFH was supported by a career development grant K01MH099141 from the National Institute of Mental Health. BTB was supported by a career development grant K08HD075831 from the National Institute of Child Health and Human Development. JMD was supported by R01 (R01DA032889-04) and R21 (R21DA041706-01) grants from the National Institute of Drug Abuse. The funding organization had no role in the design and conduct of the study; collection, management, analysis, and interpretation of the data; preparation, review, or approval of the manuscript; and decision to submit the manuscript for publication.

Competing interests: All authors have completed the ICMJE uniform disclosure form at www.icmie.org/coi disclosure.pdf and declare: SHD has consulted for AstraZeneca and UCB for unrelated topics and is co-investigator of a study funded by Eli Lilly and Pfizer, unrelated to the topic of this manuscript; $\mathrm{KFH}$ and BTB are co-investigators of a study funded by Eli Lilly and Pfizer, unrelated to the topic of this manuscript; BTB is the principal investigator of a study funded by Baxalta, unrelated to the topic of this manuscript; EP is co-principal investigator of a study funded by Boehringer Ingelheim and site principal investigator of a study funded by GlaxoSmithKline, both unrelated to the topic of this manuscript; no financial relationships with any companies that might have an interest in the submitted work in the previous 3 years; no other relationships or activities that could appear to have influenced the submitted work.

Ethical approval: The research was approved by the institutiona review board of Brigham and Women's Hospital, which granted a waiver of informed consent (IRB 2013P001741).

Data sharing: No additional data available.

Transparency: The lead author affirms that the manuscript is an honest, accurate, and transparent account of the study being reported; that no important aspects of the study have been omitted; and that any discrepancies from the study as planned (and, if relevant, registered) have been explained.

This is an Open Access article distributed in accordance with the Creative Commons Attribution Non Commercial (CC BY-NC 4.0) license, which permits others to distribute, remix, adapt, build upon this work non-commercially, and license their derivative works on different terms, provided the original work is properly cited and the use is noncommercial. See: http://creativecommons.org/licenses/ by-nc/4.0/.

1 Centers for Disease Control and Prevention (CDC). Vital signs: overdoses of prescription opioid pain relievers-United States, 1999-2008. MMWR Morb Mortal Wkly Rep 2011;60:1487-92.

2 Volkow ND. America's addiction to opioids: heroin and prescription drug abuse. Senate Caucus on International Narcotics Control. https://www.drugabuse.gov/about-nida/legislative-activities/ testimony-to-congress/2016/americas-addiction-to-opioids-heroinprescription-drug-abuse. 2014

3 Bateman BT, Hernandez-Diaz S, Rathmell JP. Patterns of opioid utilization in pregnancy in a large cohort of commercial insurance beneficiaries in the United States. Anesthesiology 2014;120: 1216-24. doi:10.1097/ALN.0000000000000172

4 Desai RJ, Hernandez-Diaz S, Bateman BT, Huybrechts KF. Increase in prescription opioid use during pregnancy among Medicaid-enrolled women. Obstet Gynecol 2014;123:997-1002. doi:10.1097/ AOG 0000000000000208

5 van Amsterdam J, van den Brink W. The misuse of prescription opioids: a threat for Europe?Curr Drug Abuse Rev 2015;8:3-14 doi:10.2174/187447370801150611184218

6 Engeland A, Bramness JG, Daltveit AK, Rønning M, Skurtveit S, Furu K. Prescription drug use among fathers and mothers before and during pregnancy. A population-based cohort study of 106,000 pregnancies in Norway 2004-2006. BrJ Clin Pharmacol 2008;65:653-60. doi:10.1111/j.1365-2125.2008.03102.x

7 Hudak ML, Tan RCCommittee On DrugsCommittee On Fetus And NewbornAmerican Academy of Pediatrics. Neonatal drug withdrawal. Pediatrics 2012;129:e540-60. doi:10.1542/peds.2011-3212

8 Patrick SW, Davis MM, Lehmann CU, Cooper WO. Increasing incidence and geographic distribution of neonatal abstinence syndrome: United States 2009 to 2012. J Perinatol 2015;35:650-5. doi:10.1038/ jp.2015.36

9 Patrick SW, Schumacher RE, Benneyworth BD, Krans EE, McAllister IM, Davis MM. Neonatal abstinence syndrome and associated health care expenditures: United States, 2000-2009. JAMA 2012;307:1934-40. doi:10.1001/jama.2012.3951

10 FDA Drug Safety Communication. Antipsychotic drug labels updated on use during pregnancy and risk of abnormal muscle movements and withdrawal symptoms in newborns. http://www.fda.gov/Drugs/ DrugSafety/ucm243903.htm. 2011

11 Carrasco M, Rao SC, Bearer CF, Sundararajan S. Neonatal gabapentin withdrawal syndrome. Pediatr Neurol 2015;53:445-7. doi:10.1016/i.pediatrneurol.2015.06.023

12 Hanley GE, Mintzes B. Patterns of psychotropic medicine use in pregnancy in the United States from 2006 to 2011 among women with private insurance. BMC Pregnancy Childbirth 2014;14:242. doi:10.1186/1471-2393-14-242

13 Hwang CS, Kang EM, Kornegay CJ, Staffa JA, Jones CM, McAninch KK. Trends in the concomitant prescribing of opioids and benzodiazepines, 2002-2014. Am J Prev Med 2016:51:151-60. doi:10.1016/j.amepre.2016.02.014

14 Desai RJ, Huybrechts KF, Hernandez-Diaz S. Exposure to prescription opioid analgesics in utero and risk of neonatal abstinence syndrome: population based cohort study. BMJ 2015;350:h2102. doi:10.1136/ bmj.h2102

15 Palmsten K, Huybrechts KF, Mogun H. Harnessing the Medicaid Analytic eXtract (MAX) to evaluate medications in pregnancy: design considerations. PLoS One 2013;8:e67405.. doi:10.1371/journal. pone. 0067405

16 Von Korff M, Saunders K, Thomas Ray G. De facto long-term opioid therapy for noncancer pain. Clin J Pain 2008;24:521-7. doi:10.1097/AJP.0b013e318169d03b

17 Mercadante S, Porzio G, Aielli F. Opioid switching from and to tapentadol extended release in cancer patients: conversion ratio with other opioids. Curr Med Res Opin 2013;29:661-6. doi:10.1185/030 07995.2013.791617

18 Mercadante S, Villari P, Ferrera P. Safety and effectiveness of intravenous morphine for episodic breakthrough pain in patients receiving transdermal buprenorphine. Pain Symptom Manage 2006;32:175-9. doi:10.1016/j. jpainsymman.2006.01.013

19 Clinical Pharmacology [database online]. Tampa, FL: Gold Standard, 2016. http://www.clinicalpharmacology.com.

20 Bateman BT, Mhyre JM, Hernandez-Diaz S. Development of a comorbidity index for use in obstetric patients. Obstet Gynecol 2013;122:957-65. doi:10.1097/AOG.0b013e3182a603bb

21 Schneeweiss S, Seeger JD, Maclure M, Wang PS, Avorn J, Glynn RJ. Performance of comorbidity scores to control for confounding in epidemiologic studies using claims data. Am J Epidemiol 2001:154:854-64. doi:10.1093/aje/154.9.854

22 Desai RJ, Rothman KJ, Bateman BT, Hernandez-Diaz S, Huybrechts KF. A Propensity score based fine stratification approach for confounding adjustment when exposure is infrequent. Epidemiology 2017;28:249-57. doi:10.1097/ EDE.0000000000000595

23 Schneeweiss S, Rassen JA, Glynn RJ, Avorn J, Mogun H, Brookhart MA. High-dimensional propensity score adjustment in studies of treatment effects using health care claims data. Epidemiology 2009;20:512-22. doi:10.1097| EDE.0b013e3181a663cc

24 Finnegan LP, Connaughton JFJ Jr, Kron RE, Emich JP. Neonatal abstinence syndrome: assessment and management. Addict Dis 1975;2:141-58.

25 Dryden C, Young D, Hepburn M, Mactier H. Maternal methadone use in pregnancy: factors associated with the development of neonatal 
abstinence syndrome and implications for healthcare resources. BJOG 2009;116:665-71. doi:10.1111/j.1471-0528.2008.02073.x

26 Jansson LM, Di Pietro JA, Elko A, Williams EL, Milio L, Velez

$M$. Pregnancies exposed to methadone, methadone and other illicit substances, and poly-drugs without methadone: a comparison of fetal neurobehaviors and infant outcomes. Drug Alcohol Depend 2012;122:213-9. doi:10.1016/j. drugalcdep.2011.10.003

27 Wachman EM, Newby PK, Vreeland J. The relationship between maternal opioid agonists and psychiatric medications on length of hospitalization for neonatal abstinence syndrome. J Addict Med 2011:5:293-9. doi:10.1097/ADM.0b013e3182266a3a

28 Rothman K, Greenland S, Lash T. Validity in Epidemiologic Studies. In: Rothman K, Greenland S, Lash T, eds. Modern Epidemiology. Lippincott Williams \& Wilkins, 2012: 128-47.
29 Raffaeli G, Cavallaro G, Allegaert K. Neonatal abstinence syndrome: update on diagnostic and therapeutic strategies. Pharmacotherapy 2017. doi:10.1002/phar.1954.

30 Norton JW. Gabapentin withdrawal syndrome. Clin Neuropharmacol 2001:24:245-6. doi:10.1097/00002826-200107000-00011

31 Mersfelder TL, Nichols WH. Gabapentin: abuse, dependence, and withdrawal. Ann Pharmacother 2016;50:229-33. doi:10.1177/1060028015620800

32 Loudin S, Murray S, Prunty L, Davies T, Evans I, Werthammer J. An atypical withdrawal syndrome in neonates prenatally exposed to gabapentin and opioids. J Pediatr 2017;181:286-8. doi:10.1016/j. peds.2016.11.004

Appendix: Supplementary tables A-M, figures A-B, and case report 